Methods: A total of 426 newly diagnosed RA patients treated from August 2011 to December 2014 were included. The DAS28-P score (the subjective components of the DAS28 relative to the total components) was calculated as DAS28-P = $0.56^{*}$ sqrt(TJC28) $+0.014^{*}\left(\right.$ VAS-GH) $/ 0.56^{*}$ sqrt(TJC28) $+0.28^{*}$ sqrt(SJC28) + $0.78 \ln (E S R)+0.014^{*}($ VAS-GH). The EULAR response was assessed after 6 months of treatment. Of those who failed to attain good EULAR responses, those for whom the objective measures (the ESR, the CRP level, and swollen joints) were normalized were defined as having failed treatment because of subjective measures.

Results: The mean age of all patients was 54 years and $79 \%$ were female. The median (IQR) DAS28 score at baseline was $4.8(4.04-5.49)$ and that after 6 months of treatment 3.21 (2.41-3.95). Good responders (according to the EULAR criteria) numbered 180 (38.9\%), moderate responders $150(32.4 \%)$, and non-responders $96(20.7 \%)$. The DAS28-P score fell significantly from baseline to 6 months in good $(0.43$ versus $0.28, p<0.001)$ and moderate responders $(0.44$ versus $0.4, p=0.003)$, but not in non-responders $(0.43$ versus $0.45, p=0.727$ ) Younger age, a lower DAS28 score, and a lower DAS28-P score at baseline were related to a good EULAR response. Subjects who failed to respond because of subjective measures tended to have higher DAS28-P scores at baseline.

Conclusions: We found that RA patients with high DAS28-P scores, reflecting subjective measures, were less likely to achieve good EULAR responses 6 months after treatment initiation and tended not to be classified as good responders despite normalization of objective measures.

Disclosure of Interest: None declared

DOI: 10.1136/annrheumdis-2017-eular.1588

\section{FRI0104 NO ADDED PREDICTIVE VALUE OF SERUM CALPROTECTIN FOR TREATMENT RESPONSE TO ADALIMUMAB OR ETANERCEPT IN RA PATIENTS}

L. Tweehuysen ${ }^{1}$, N. den Broeder ${ }^{1}$, L.A.B. Joosten ${ }^{2}$, T. Vogl ${ }^{3}$, F.H.J. van den Hoogen ${ }^{1,4}$, R.M. Thurlings ${ }^{4}$, A.A. den Broeder ${ }^{1,4}$. ${ }^{1}$ Rheumatology, Sint Maartenskliniek; ${ }^{2}$ Internal Medicine, Radboudumc, Nijmegen, Netherlands; ${ }^{3}$ Immunology, University of Münster, Münster, Germany; ${ }^{4}$ Rheumatology, Radboudumc, Nijmegen, Netherlands

Background: Approximately $60 \%$ of RA patients do not achieve good clinical response after 6 months of treatment with a TNF inhibitor (TNFi). ${ }^{1}$ To date, no clinically useful baseline biomarkers have been found to predict response. ${ }^{2}$ Calprotectin (a heterodimer of S100A8/S100A9) was shown to be modestly predictive for treatment response to adalimumab (ADA) (area under the curve (AUC): 0.69) while no difference in calprotectin levels was found between responders and non-responders in RA patients treated with etanercept (ETN) and methotrexate ${ }^{3,4}$

Objectives: To assess the added predictive value of serum calprotectin for clinical response after 6 months treatment with ADA or ETN in RA patients.

Methods: RA patients starting treatment with ADA or ETN in the BIO-TOP study (a prospective cohort study) were included. Patients who discontinued TNFi treatment within 2 months were excluded from analysis. Serum calprotectin was measured at baseline using ELISA. EULAR response was measured at 6 months (good versus moderate/no response). Discontinuation of TNFi before 6 months was regarded as non-response (in case of lack of effect) and clinical response at 3 months was carried forward (when stopped for other reasons). First calprotectin levels were correlated cross-sectionally with several clinical baseline markers. Thereafter receiver-operator-characteristic $(\mathrm{ROC})$ curves were created for ADA and ETN separately. Finally logistic prediction models (basic model and calprotectin added model) were created using backward selection, including baseline characteristics and calprotectin levels to examine the added predictive value of calprotectin.

Results: Data on calprotectin levels and EULAR response were available for 125 patients (ADA $(n=50)$, ETN $(n=75))$ with $40 \%$ of patients achieving EULAR good response. Responders showed significantly higher calprotectin levels at baseline: $985 \mathrm{ng} / \mathrm{mL}$ (p25-p75: 558-1417) versus $645 \mathrm{ng} / \mathrm{mL}$ (p25-p75: 415973) $(p=0.04)$. Calprotectin levels were significantly correlated to DAS28-CRP (Spearman $\rho=0.32, p<0.01$ ) and $\mathrm{C}$-reactive protein (CRP) levels (Spearman $\rho=0.57, p<0.01)$ and significantly higher in rheumatoid factor positive patients $(\mathrm{p}=0.03)$. No significant correlation was found between calprotectin and age, gender or ACPA positivity. The AUC for serum calprotectin in the ADA and

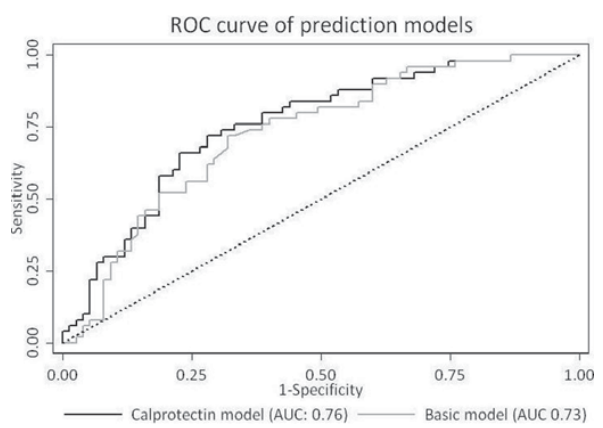

ETN group were $0.68(95 \% \mathrm{Cl}: 0.49-0.88)$ and 0.49 (95\% Cl: $0.35-0.63)$, respectively. The basic model (backward selected variables: baseline DAS28CRP and medication used (ADA versus ETN)) showed an AUC of $0.73(95 \% \mathrm{Cl}$ : 0.64-0.82). The calprotectin added model performed similarly with an AUC of $0.76(95 \% \mathrm{Cl}: 0.67-0.84)(\mathrm{p}=0.27)$.

Conclusions: Serum calprotectin is modestly predictive for EULAR good response to ADA but not ETN treatment after 6 months in RA patients. However, calprotectin does not provide additional predictive value over a basic clinical prediction model.

References:

[1] Hetland et al. Arthritis Rheum 2010;62(1):22-32.

[2] Cuppen et al. Rheumatology (Oxford) 2016;55(5):826-39.

[3] Choi IY et al. Ann Rheum Dis 2015;74(3):499-505.

[4] Obry A et al. PLoS One 2014;9(12):e115800.

Disclosure of Interest: L. Tweehuysen: None declared, N. den Broeder: None declared, L. Joosten: None declared, T. Vogl: None declared, F. van den Hoogen Consultant for: Biogen, Celltrion, Janssen, Mundipharma and Sandoz, R. Thurlings: None declared, A. den Broeder Consultant for: Amgen

DOI: 10.1136/annrheumdis-2017-eular.3751

\section{FRI0105 FLUCTUATING, UNPREDICTABLE AND CHALLENGING: HOW PAIN, FATIGUE AND SLEEP DISTURBANCE IMPACT QUALITY OF LIFE IN PEOPLE WITH RHEUMATOID ARTHRITIS}

V. Short ${ }^{1}$, J. McBeth ${ }^{1}$, K.L. Druce ${ }^{1}$, S. Moore ${ }^{2}$, W. Dixon ${ }^{1}$, S. Kyle ${ }^{3}$, M. Lunt ${ }^{1}$, L. Cordingley ${ }^{4} .{ }^{1}$ Arthritis Research UK Centre for Epidemiology, University of Manchester; ${ }^{2}$ Research User Group, NIHR Manchester Musculoskeletal

Biomedical Research Unit, Central Manchester University Hospitals NHS Foundation Trust, Manchester; ${ }^{3}$ Sleep \& Circadian Neuroscience Institute, Nuffield Department of Clinical Neurosciences, University of Oxford, Oxford;

${ }^{4}$ Division of Musculoskeletal and Dermatological Sciences, University of Manchester, Manchester, United Kingdom

Background: Qualitative studies of patient experiences have provided useful insights into how rheumatoid arthritis (RA) affects quality of life (QoL). RA threatens self-image and feelings of independence, reduces personal control, and is associated with difficulty in juggling self-management alongside pre-existing demands of work, home and family life. Pain, fatique, sleep problems and low mood are commonly reported by patients and perceived as particularly troublesome aspects of RA. We wanted to know whether these symptoms of RA were viewed as the key drivers of reported changes to QoL.

Objectives: To explore RA patients' perceptions of RA-related pain, fatigue, sleep problems and mood, and their impact on QoL.

Methods: Participants, recruited through RA charities across England, were purposively sampled to include a broad range of current ages and ages at onset of RA. Eleven individuals with RA were interviewed either in a group interview (three participants) or individual semi-structured interviews (eight participants). The interview topic guide was developed in consultation with patient representatives. Interviews were audio-recorded and transcribed in full. Interview data were analysed using inductive latent thematic analysis.

Results: All participants were female, aged $36-75$ years. Disease duration ranged from five months to 31 years.

Pain, fatigue, poor sleep and low mood were all attributed to RA, and viewed as having wide-ranging impacts on QoL. Participants saw the bidirectional nature of relationships between these four symptoms as particularly challenging. For example, reduced sleep led to increased pain and vice versa.

Themes included: "Fluctuating symptoms" referring to the impact of the significant changes in mood, fatigue or pain which can occur within a single 24 hour period during "an RA flare"; "The unpredictability of symptoms" was viewed as emotionally draining, anxiety-provoking and undermined both short and long-term plans; "Challenging" described both the symptoms and the need to anticipate potential daily challenges in the context of current levels of symptoms.

Most were able to articulate the attitudinal, practical and emotional strategies which could facilitate adaptation to living with RA such as the need to maintain optimism, a focus on what could realistically be achieved, day-to-day cognitive flexibility and practical support.

Pain, fatigue and changes to sleep patterns were viewed as affecting the following aspects of QoL; ability to work effectively, family and social relationships, self-worth, ability to feel "in control", threats to future plans and appearance. The appearance theme included a desire not to be viewed as ill or "disabled", weight-gain and self-consciousness.

Conclusions: Daily and hourly variations in pain, fatigue and low mood are challenging for individuals with RA. The unpredictable nature of RA often was associated with anticipatory anxiety and mood fluctuations within the day. However, despite this the importance of positivity and acceptance were identified as mitigating some of the impact RA was having on QoL.

Acknowledgements: This study was funded by an Arthritis Research UK project grant (Project number 21188)

Disclosure of Interest: None declared

DOI: 10.1136/annrheumdis-2017-eular.1849 